\title{
COMPLEMENTARY MOLECULAR MODELS OF LEARNING AND MEMORY
}

\author{
MICHAEL CONRAD \\ Department of Computer and Communication Sciences, University of Michigan, Ann Arbor, MI 48104, U.S.A.
}

(Received February 17th, 1976)

(Revision received August 3rd, 1976)

\begin{abstract}
The functional capabilities of the brain are formally characterizable in terms of a finite system along with a memory space which it can manipulate. Two types of learning are possible: (1) modification-based learning, associated with alternate realizations of the finite system; (2) memory-based learning, associated with the assimilation, manipulation, and retrieval of memories. Constructive models which fulfill these conditions and which at the same time operate on the basis of molecular information processing principles have certain general features. We describe these features in terms of two interfaced submodels, the first for the finite system and the second for the memory space. The finite system may be realized by networks of neurons in which the specificity of enzyme molecules controls the nerve impulse. Such a realization is amenable to modification-based learning mediated by processes analogous to those of naturai evolution and selective theories of antibody synthesis. The memory space is realizable by networks of neurons in which the conformation of dendritic receptor molecules controls the nerve impulse. In this case certain neurons firing in response to an external input undergo sensitization at the dendrites and in such a way that they are loadable and later callable by reference neurons, thereby allowing for reconstruction or manipulation of the firing pattern associated with this input. The overall construction makes a large number of biochemical, anatomical, physiological, and psychological predictions which are either testable or in good agreement with fact.
\end{abstract}

\section{Introduction}

The third quarter of the twentieth century has seen dramatic advances in both mólecular biology and in the information sciences. The biologists, on the one hand, have elucidated the fundamental mechanisms of heredity and reproduction. The information scientists, on the other, have developed concrete computing and communication devices, along with general theories about the design, limits, and capabilities of such devices. The extent to which these two developments influenced one another is a historical question, one which we cannot discuss here (cf. Burks et al., 1974). The connection itself, however, is of more than historical irsterest: for the mechanisms of heredity and self-reproduction are without doubt the fundamental mechinisms of information transfer and accumulation in nature.
The deep connection and complementarity of molecular biology and information science canno but raise the hope that the concepts in these two fields, once joined, could lead to an understanding of the biological information processor par excellence, viz. the higher nervous system and brain. Indeed, there is increasing, though highly controversial evidence that individual molecules do play a crucial role in learning and memory (Cohen, 1970; McConnell and Shelby, 1970; Hýden and Lange, 1971; Ungar, 1973). The amount of experimental work in this area is extensive and in many cases extremely sophisticated. But what seems to be lacking is an equally sophisticated use of the machinery of aformatior science to interpret these experiments; or, more apropos, any equal attempt to develop information science concepts specifically suited to describing information processing in molecular biological systems. 
One difficulty is undoubtedly that the brain is complicated. Its behavior is too intricate to describe in detail, at least in each individual case; but the details are too interesting to wash away with statistics. What can be done, however, is to characterize the capabilities of the brain, e.g. its capabilities for computing some class of functions (in the mathematical sense). Then we can construct models with these capabilities which operate on the basis of information processing principles derived from molecular biology. In order to operate on a natural, economical basis such models must have certain very general design features. These necessary design features are thus predictions about the brain.

We begin with a review of the experimental work in the area; characterize the capabilities and the information processing principles; then consider the models and their predictions.

\section{Types of experiment; types of theory}

The experimental work on the relation between macromolecules and brain function is of course enormous (for book length discussions, see, e.g. John, 1967; Byrne, 1970; Ungar, 1970; Gibbs and Mark, 1973). However, it is perhaps useful, at the very beginning, to classify this work on the basis of the type of relation which is being studied.

(i) Molecular biology of nerve cells. These studies are directed towards elucidating the characteristics of nucleic acid and protein synthesis in nerve cells (e.g., Hýden, 1967a; Hýden and Lange, 1971), the transport of materials in the cells (e.g. Weiss, 1970), and more generally the structure and function of the nerve cell and its constituents at the molecular level (cf. Schmitt, 1970).

(ii) Biochemical correletes of learning and memory. These studies are directed towards elucidating changes in nucleic acid and protein during the processes of memory acquisi- tion, consolidation, and learning. Many of the studies have been based on tracer techniques (e.g. Glassman, 1969); others on the effect of various pharmacological agents on long and short term memory (e.g. Agronoff et al., 1966, 1967; Barondes and Cohen, 1967; Agronoff, 1970; Barondes, 1970). Such experiments involve a combination of biochemical, physiological, and psychological technique.

(iii) Transfer and specific protein studies. These studies have been directed, in the first case, to the possibiisty of transferring acquired information from one part of the organism to the other or from one part of the brain to the other by transferring specific chemical agents, primarily nucleic acids (e.g. Albert, 1966; McConnell, 1970; McConnell et al., 1970). In the second instance they have been directed to determining whether changes in protein specificity accompany learning or memorization (Hýder 1967b) and also whether specific protomis are capable of effecting specific behavioral changes (Ungar, 1973). Transfer and specificity studies have been the most controversial and difficult to interpret aspect of biochemicall and molecular neurobiology (cf. Byrne et al., 196:)

The theoretical work on the aree has perhaps been even more diverse than the experimental work. Thus we must also classify this (again at the expense of great simplification) on a typological basis.

(i) Regulative theories. What might be called regulative theories hold that what changes in learning and memory are the regulatory properties of nerve cells, e.g. the quantity of chemical entities available for synaptic transmission (e.g. Bennett et al., 1970), or the amount of synthetic machinery in the cell (cf. Lehninger, 1970). These theories do not assign any specific role to nucleic acid and protein, but only claim that changing characteristics of the nerve cell have a molecular basis. A seeming implication of such theories is that the learning and memory processes could be understood in terms of networks of 
modifiable nerve cells, the major contribution of the molecular description being to understand the underlying basis of the modifications. It should be pointed out that some workers in the field feel that all the molecular and biochemical correlates of learning are incidental byproducts or prerequisites of functions which can be understood, or at least described, solely at the cellular level.

(ii) Specificity theories. What might be called specificity theories make the claim that specific molecular changes are concomitant to learning and memory. There are a number of such theories, including theories based on enzyme induction (e.g. Smith, 1962), on the relationships between specific proteins and particular dendritic pathways (e.g. Ungar, 1973), on the developmental specificity of the nervous system (e.g. Mark, 1974), to mention only a few. There are also a number of theories which hypothesize some kind of reverse transcription, i.e. storage of information in nucleic acid sequence.

(iii) Conformational theories. What might be called conformation theories are based on the idea that nerve impulses are capable of causing conformational changes in membranes (e.g. McConnell, 1970), protein molecules (Neumann, 1974; Rosen, 1974), or nucleic acids (Katchalsky and Neumann, 1973), and that information is stored in these conformational changes.

The above brief survey is necessarily sketchy and selective. We should also point out that regulative, specific, and conformational models are not mutually exclusive, and also that all molecular models must be interfaced with the paramount fact of the electrical activity of nerve cells. Indeed, it is this interfacing which is the main difficulty (cf. Stent, 1972).

3. Information sience and molechlar information science

Many models of learning and memory are based on ideas drawn from network, auto- mata, computer theory, and artificial intelligence (cf. Conrad et al., 1974). In general, however, these models derive from our experience with conventional information processing devices, and therefore ignore and are not even suited to deal with the molecular nature of biological systems. A better way to develop such models is to start with the already existing concepts of automata and computer theory, but then to alter them so that they are suitable for dealing with molecular processes, taking as an assumption that the brain is in fact a molecular information processing device (Conrad, 1972b, 1973a, 1974f).

The simplest formalization of automata and computers and the one which has had the most impact on hrain theory is the Turing scheme (or machine, cf. Fig. 1). This is a finite automaton (or system with finite sets of states, inputs, and outputs plus state and output transition rules) along with a tape which it can mark and move. Such a scheme is universal if it can read and follow any particular rule encoded in the sequence of markings on the tape. Any general purpose computer is universal in this sense.) Also, the scheme may include some abstraction of the environment, so that the inputs is the automaton include both the markings on the tape and the states of the environment.

What makes the Turing scheme particularly interesting is the picture which apparently motivated its formulation (Turing, 1936; Minsky, 1967). Turing imagined a person working an arithmetic problem on a note pad. The person can read any symbol on the pad, rewrite this symbol according to his state of mind, move to a neighboring square on the pad, and change his state of mind according to some rule. The Turing scheme is no more than a formalization of this picture, i.e. the formalization of a psychological process. Moreover, it characterizes the widest class of processes which the brain could reasonably be expected to execute in an algorithmic way; for, so far as is known, any computable function is computable within the framework of the scheme (cf. Davis, 1958). 


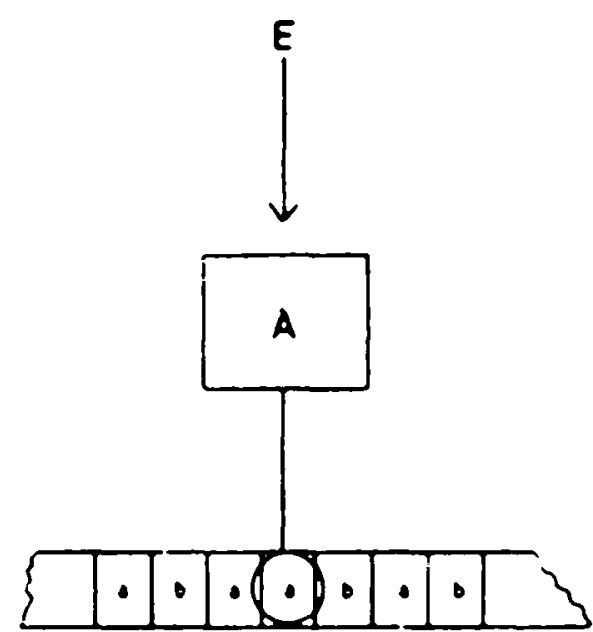

Fig. 1. Turing sheme. The echeme consists of an automaton, A, abong with a tape which it can mure and overwrite. Small letter (a and b) are lape sym. bols and $E$ thn extermal environment. The automaton reads the tape symbol, either rewrites this symbol or moves to a new tape position, and changes its state accordingly. By edaing an external environment we allow it to recond external events and include the pos. sibility of adaption to the outuide world. Memory. besed learning involves use of the tape for recording and manipulating information. In modification-band learning the ruk coverning the next state and output behavior of the automaton itself changes, i.e. we replace $A$ by $A^{\prime}$. The Turing mechine is a procese, not a real machine. Its importance lies in the simplicity with which it formalizes paycholocical processes, and the completenes with which it formalizes algorithmic procesen. With the addition of the external environ. ment we can also formalize nonatgorithmic information proceses. vix. evolutionary procreses. The Turing scheme thus provides conceptual boundery conditions for constructing concrete brain models.

Not all information processes are algorithmic, howevor. For example, evolution based on chance variation and natural selection is certainly of a quite different nature. Thus we cannot assume that all psychological processes are algorithmic. Nevertheless, we can still characterize these processes within the frame. work of the Turing scheme; for this scheme remains a valid abstraction of psychologica! precesses whether or not these are algorithmic (Conrad, 1974d).

The main psychological process we are interested in is learning, which roughly speal:ing we can regard as change in a system's behaviour which either makes that behavior more adapted to the piven environment or allows it to adapt to novel envronments. The Turing formalization allows for three types of leaming:

(1) Inherent capability of the automaton. In this case the leaming is based on the computational capability of the automaton, but without using the tape. For example, the automaton may be so designed that interac. tion with the environment drives it into a state appropriate to the future behavior of the environment.

(2) Memory-bosed learning. In this case the automaton is allowed to use the tape. For example, it may record environmental events on the tape and use these records to control its future behavior; or it may use the tape to increase its problem solving power. Memory. based learning includes programmability from input (if the automaton is universal and a more suitable rule is encoded on its input tape).

(3) Modification-based learning. In this case automaton $A$ is replaced by automaton $A$. where $A^{\prime}$ is more ${ }^{\prime}$ to the environment.

Types (1) and (2) are both basically atg(1. rithmic (in the sense that definite rules are being (ollowed). Actually, both involve memory and in principle differ only if the tape (storage area) is in some way potentially unbounded. From the practical standpoint, how. ever, it is to be expected that the dirtinction would retain validity even in the finite case. Type (3) is not algorithmic since it involves the raplacement of one process by another.

The above point is critical. The Turing scheme, no matter how convenient as a formalism, is just that, viz. a process and not a real machine. The problem of brain modeling is thus to construct realizations which on the one hand have all the functional capabilities of the brain (e.g. as formalized with Turing schemes) and at the same time operate on the basis of biological principles and generate predictions which conform to the facts. In the case of type (3) iearning the realization changes, but not according to the rule which 
is embodied in the realization (as opposed to e.g. chance variation and selection).

There are, of course, many concrete realizations which have sufficient functional capabilities (essentially all digital computers). All of these share one fundamental feature in common. They are built out of a finite set of fixed components (or canonical automata) which are linked together and set appropriately so that they embody any particular rule. Moreover, this linkage and setting process may be done in a definite, algorithmic way (see, e.g., Conrad and Dal Cin, 1972). The most well-known example, from the standpoint of neuroscience, are networks of formal neurons, such as the McCulloch-Pitts networks (McCulloch and Pitts, 1943; Arbib, 1965; cf. Fig. 2). These are, of course, the extreme abstractions of real neurons, i.e. components which fire when the sum of their
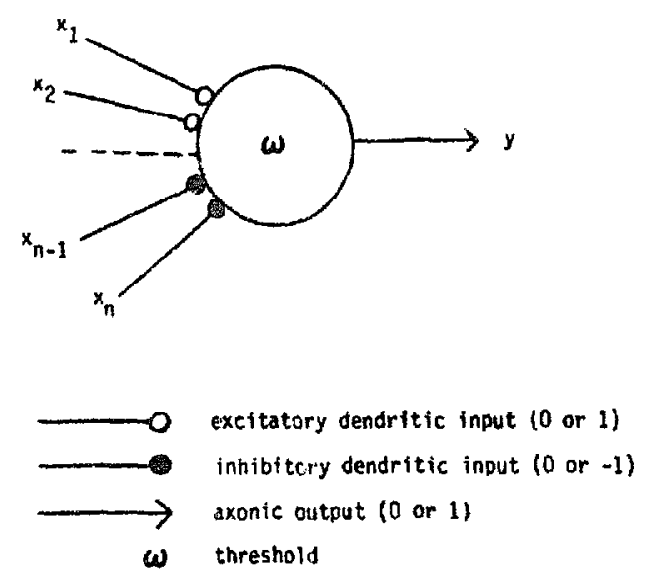

Fig. 2. McCulloch-Pitts formal neuron. The neuron fires when the sum of the excitatory and inhibitory inputs exceeds the threshold. The automaton of the Turing scheme can always be simulated by a properly constructed network of formal neurons. The importance of the McCulloch-Pitts model is that it shows that one can embody the most complex automata behavior in networks of neurons which are the extreme abstraction of real neurons. However, such networks are generally unecenomical in terms of number of neurons and inamenable to gradual transformation of function with gradual transformation of structure. "This is generally true for structurally programmable systems, i.e. systems in which the transition functions are encoded in the network connectivity and initial state of a set of canonical components (or canonical base automata). excitatory and inhibitory inputs exceeds some threshold. Indeed, this is why the McCulloch-Pitts model has played such an important role in brain theory; it showed, for the first time, that one could in principle do all one needed (except for memory manipulation) with what McCulloch called the most impoverished version of a neuron (cf. Rosen, 1969).

I will call systems of the above type strueturally programmable (since the rule which generates their behavior is encoded in the network structure and initial automata settings in an effective way). The difficulty with structurally programmable systems is that they are very uneconomical in comparison to the brain (in terms of number of elements, cf. Minsky, 1967) and therefore uneconomical as regards processes of type (1); but even more important, they are not amenable to gradual transformation of function with gradual transformation of structure (Conrad, 1973a, 1974a), and therefore do not allow for learning processes of type (3) (Conrad, 1972a; cf. also Minsky, 1961; Bremermann et al., 1966). Furthermore, formalized nerve network models do not incorporate a tape ( $O x$ memory space) device in any natural way, and therefore exclude learning processes of type (2).

These difficulties are not necessary, however. In fact, one can construct structurally nonprogrammable computers with general powers of computation which at the same time conform to the histological structure of the brain. These models are based on an alternate, more general concept of information processing in which we modify the rule executed by the system by modifying the functions performed by the elementary components rather than by modifying the interlinkage of these components (Conrad, 1974b, c). For example, imagine that we want a contractor to build houses suitable for different conditions. In the conventional picture we would give him a certain canonical set of builuing blocks and different blueprints. In the new picture he would ase the same blueprint but we would supply different building blocks. This picture, in fact, is the fundamen- 
tal basis of evolution, since genetic changes ultimately appear as changes in the primary structure and therefore function of protein. Furthermore, because of the folding properties of proteins slight changes in primary structure are often concomitant to only slight changes in three dimensional structure and therefore function, thus allowing the organism to adapt to the environment in a step by step fashion.

\section{Molecular control of the nerve impulse}

\subsection{The selection circuit model}

Now we can turn to the properties which the brain would have to have in order to support gradual transformation of funstion on the basis of the molecular process indicated above (and therefore modification-bas?d learning) and at the same time support powers of computation equivalent to that of a finite automaton. The basic idea is that individual molecules - the components amenable to gradual transformation of function - control the nerve impulse. To this end we introduce a more complex formalization of the neuron, one which captures the geometrical asymmetries and membrane nonlinearities of the real neuron. In this new formalization (to be called the enzymatic neuron, see Fig. 3) the effect of each dendritic input at any particular locus on the cell surface is determined by a weighting function which depends on the geometry of the neuron, the conductivity properties of the membrane, and on the activity of other dendrites. The neuron also carries populations of molecules or molecular aggregates (to be called excitases) which catalyze events leading to impulse formation under suitable degrees of excitation. Thus, the enzymatic neuron fires if the pattern of dendritic inputs produces a suitable degree of excitation at any locus which is in fact occupied by such an excitase.

The question arises: how can the brain know in advance (and witho it computation,

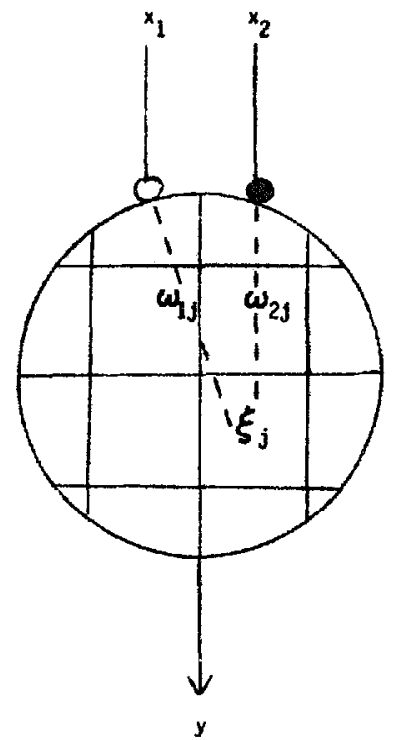

$\longrightarrow$ excitatory dendritic input $\left(x_{i}=0\right.$ or 1$)$
$\longrightarrow$ inhibitory dendritic input $\left\{x_{i}=0\right.$ or -1$)$
$\omega_{i j}=$ axonic output $(y=0$ or 1$)$
$\xi_{j}=$ excitase molecule in region $j$

Fig. 3. Enzymatic neuron. The ieuron fires whenever the weighted sum of the excitatory and inhibitory dendritic inputs (of which only two are shown) excites a region of the cell surface at which excitase enzymes are located. The weighting coefficients make it possible to work with formal neurons which reflect the complex morphological and conductivity properrties of real neurons. In principle each such coefficient could also be written as a function of the pattern of dendritic firing itself. The particular patterns of firing to which the neuror responds depends on the excitase molecules which it contains. By adding or deleting excitase molecules which bind at different places on the cell surface it is possible to increment or decrement this set of patterns by a single member and therefore to gradually modify the function performed by either individual enzymatic neurons or networks of enzymatic neurons (independence property). In general such networks are very efficient, because each neuron is a multithreshold element and therefore much less of a gross averaging device than the MeCul. loch-Pitts neuron (cf. Fig. 2). Enzymatic networks are not structurally programmable because they are not constructed from a canonical set of components which can be linked together in different ways to porform different functions. Instead the components themselves are changed, by changing the complement of excitases which they carry. 
which is infeasible) which excitases will do the job (e.g. locate themselves in the right places)? The unly possibility is by trial and error evolution, analogous to evolution by variation and natural selection. But this is possible only if the brain contains some kind of artificial selection system which tests and evaluates excitase' species and (on the basis of this evaluation) stimulates the production of transforming nucleic acid which code for these species. Furthermore, the tissue transformed must be essentially interchangeable with the original tissue, otherwise the weighting coefficients would be different and the favored excitases would not have the same function (cf. Fig. 4). Thus the sine qua non properties of the model are:

(i) Redundancy. The brain consists of various types of local networks of which there are many interchangeable replicas of each type.

(ii) Specificity. Each local network contains (enzymatic) neurons whose firing is controlled by excitase molecules. These enable the neuron to fire in response to specific patterns of inputs, e.g. to be a multithreshold element. Each such excitase molecule (or molecular aggregate) is capable of binding at a specific region of the cell surface, and cata. lyzes events leading to impulse formation if the particular pattern of inputs results in a high degree of excitation at that point on the cell surface.

(iii) Selection circuits. The brain contains circuits which test and evaluate the function of local networks and which control the pro. duction of culturable excitases on the basis of this evaluation. The nucleic acids whose production is stimulated diffuse out of the local ne: works in which they are produced to transform adjecent networks of the same type (in the fashion of an RNA virus). The function of the excitase molecules is the same in tinese new networks because the tissue structure and cellular properties are the same (redundancy

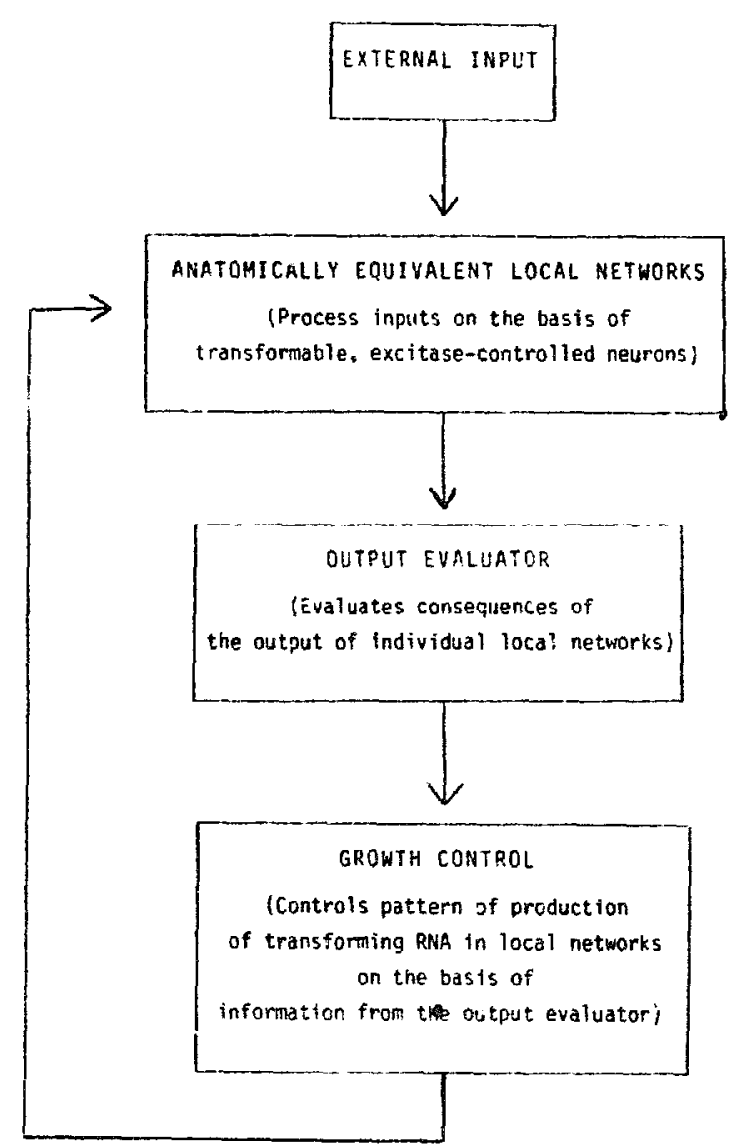

Fig. 4. Flow of information in the selection circuit model. The basic idea is that the brain contains a system for selectively culturing nucleic acids which code for molecules (excitases) capable of controlling the nerve impulse (cf. Fig. 3). External inputs are processed independently by local networks which are essentially interchangeable as regards connectivity and weighting coefficients of corresponding neurons. The output of each such local network has consequences for the organism, e.g. in terms of pleasurable or pain tul experience. The output evaluator assigns a fitness value to each local network on the basis of these consequences and transmits this information to the growth control, which in turn transmits signals back to the local networks which either stimulate or inhibit the production of exportable RNA capable of transforming neighboring networks. Advantageous exiitases associated with especially fit local networks (or tisiues) thus spread to neighboring networks, where they have the same function because of the interchingeability property. The selection circuit scheme accounts for modification-based learning, not learning based on rapid acquisition of memories or manipulation of memories. It is much more efficient than natural evolution because the selection circuits (i.e. evaluation and growth control) allcw for very iatense selection on the basis of arbitrarily small dif. ferences in fitness. 
property). The culturable excitase "genes" either pre-exisi in small numbers, in which case they (but not their distribution) are inherited (germ line type theory); or they arise from somatic processes, in which case they are not heritable at all (somatic mutation type theory); or from a combination of germ line and somatic mechanisms.

The operations of the selection circuits require certain control (e.g. inducible) enzymes. The distinction between control and excitase enzymes is important for interpreting experiments on protein changes accompanying learning (in particular the experiments of Hýden, 1967b, cf. section 5). The selection circuit model makes a large number of predictions about the brain, including predictions about the conditions under which chemical transfer of learning is possible, the effects of ablation, the specificity of brain structure, the dynamical properties of neurons (Conrad, $1973 \mathrm{~b}, 1974 \mathrm{~b}, \mathrm{~d})$. These are also described in a later section, after we pay proper attention to the problem of memory. Here, however, we $t$ : ist point out the deep analogy between tho ory and selective theories of antibody pro suction and immunity (cf. Jerne, 1955). We also point out that the number of excitase molecules which such a system can try out in the first twenty years of life is enormous - in fact, with only mild assumptions it comes to much more than all the genomes that could have been tried out in human evolution (cf. Conrad, 1974d).

\subsection{Conformationai model of memory and memory-based learning}

It is fairly simple to show that networks of excitase based neurons have all the computational capabilities of conventional formal neural nets (and therefore finite automata), but at the same time are amenable to gradual tranformation of function (Conrad, 1974d; cf. also. Schwabauer, 1976). After all, the McCulloch-Pitts networks are just a special case of a more general enzymatic network.
However, the model does not allow for computing as wide a class of functions as a Turing system. This is because it does not account for memory acquisition, manipulation, and retrieval, or for forms of learning in which it is potencially possible to consummate the learning process in a single trial (e.g. classical conditioning or instrumental learning, cf. Miller, 1967). Thus it cannot account for process (ii), i.e. memory-based learning. The latter is the type of learning for which present day computers are most suited and on which virtually the whole field of artificial intelligence is based (cf. Feigenbaum and Feldman, 1963; Bremermann, 1973; Josephsor, 1974).

The main difficulty is that the computer has an addressable memory, i.e. each bistable element can be accessed and switched by specifying its location in an array (cf. Fig. 5a). This makes it possible to avoid mixing up (or superimposing) memories because each element is uniquely accessed by activating two lines. This simple scheme is not economical and in fact not even feasible in networks of neurons (because of their converging inputs and diverging outputs, cf. Fig. 5b). Nevertheless, there is compelling evidence that the brain, like the computer, does not suffer serious problems of memory superposition.

There are a number of schemes which satisfy the above requirements, but the following is the simplest and gives the most direct account of the psychological, physiological, anatomical, and biochemical facts (Conrad, $1974 \mathrm{e}, 1975 \mathrm{a}, \mathrm{b})$.

(i) Certain neurons (to be called primaries) undergo sensitization at the dendrites when firing in response to external inputs;

(ii) The sensitized primaries are modified ("loaded") by other neurons (to be called reference neurons) and in such a way that they fire in response to the future firing of these reference neurons.

The firing of a reference neuron reconstructs the original pattern of primary activity because it only fires those neurons for which dendritic connections were opened by the loading process (see Figs. 6 and 7). The prob- 


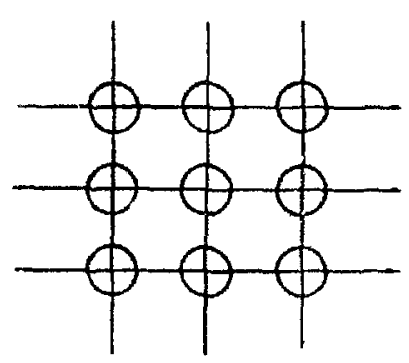

(a)

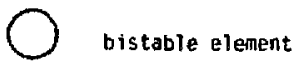

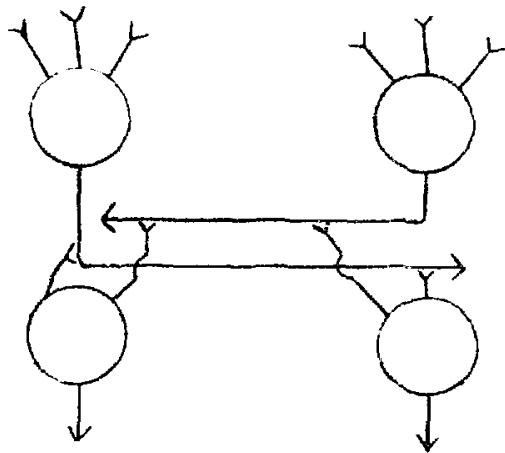

(b)

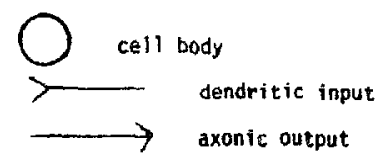

Fig. 5. Superposition problem. In an addressable computer memory (a) each bistable e'ement is uniquely accessed and switched by activating two lines. There is no problem of superposition (admixing new and old memories) because information can be stored and retrieved from individual elements without switching other elements. This is not possible in highly interconnected neural networks (b), since in general no single neuron is uniquely accessed and "switched" by activating two axonic inputs. In this case the states of the network are associated with patterns of neural firing and the problem of memory is to retrieve such patterns under appropriate circumstances, e.g. by altering dendrites in such a way that the pattern can be reconstructed in the absence of the original input. In general, however, alterations associated with new memories cannot be separated from those associated with old memories, resulting in superposition. For a simple solution to the superposition problem see Fig. 6.

lem of memory superposition is avoided because each reference neuron loads and "calls" at most one pattern of primary activity. These patterns may be arbitrarily complex (e.g. associated with complete scenes) with no extra requirement $c n$ the number of reference neurons. Moreover, if the reference neurons are activated for loading in temporal sequence, the memory structure may be ordered according to time. If primaries are also capable of loading reference neurons, these can be activated by content, e.g. by a small part of original scene. Also, the scheme allows for rememorization on the basis of the same mechanism as memory acquisition and retrieval. This is important because rememorization allows for general powers of memory manipulation (e.g. recombination of different memories, development of associative mem. ory structures, including associative structures: associated with classical conditioning and instrumental learning).

The question naturally arises: what controls which reference neurons are active at any given time and whether they will be activated for loading or caliing? This corresponds, in the case of any realization of the Turing scheme, to the question: what controls the movement of the Turing automaton on the tape and what it does to the tape? The answer here is that this is deternined by the transition functions built into the automaton. In the case of the reference neuron scheme, the transition functions must also be built into what corresponds to the automaton, e.g. into the erzymatic neural nets, except that in this case the decision process is much more complicated. Since we know that any transition functions can be built into such networks we know that the system is capable of solving the supervision problem so long as this problem is solvable. Since the brain must contain either software or hardware for such supervision, we include this function explicitly in Fig. 7 .

The reference neuron schene requires a considerable amount of neural tissue. This is materially reduced if the system utilizes party line organization (cf. Fig. 8a), in which case 


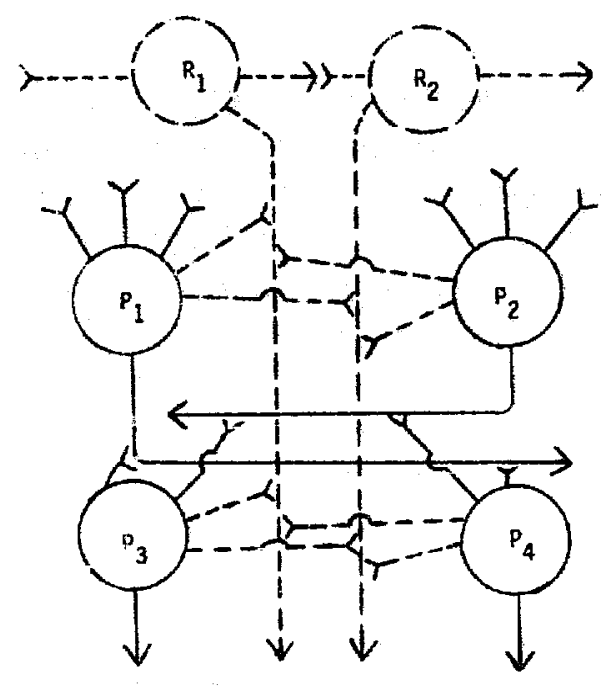

F g. 6. Leference neuron soln! iun to the superposi$t i$ in problem. The netrurk the came as the network of Fig. 5th;, except that reference neurons are a:lded. A:! new axonic ovt puts and dendritic inputs -: diatinguished by dotted lines and the neurons are labeled $\left(R_{i}=\right.$ reference neuron $i, P_{j}=$ primary neuron j). When primaries fire in response to an external input their dendrites become sensitized, i.e. assume a condition which allows them to be opened up by the reference neuron which fires immediately thereafter. In this case we say that primaries firing in response to the external input are loaded by the reference neuron and that the later firing of this reference neuron results in a call to these primaries. For exarraple, suppose that the external input results in the firing of $P_{1}$ and $P_{4}$ and that $R_{1}$ fires immediately thereafter. Then $R_{1}$ loads and is capable of later calling $P_{1}$ and $P_{4}$. There is no problem of memory superposition because each reference neuron is associated with at most one memory. The complexity of the stored memory (i.e. the number of primary firings it involves) is limited only by the number of reference to primary contacts (see Fig. 8) and the memory capacity by the number of available reference neurons. Reference neurons may activate reference neurons, in which case memories are stored and retrieved in temporal order, or they may be loaded by some of the primaries which they themselves load. Such primary to reference contacts (not shown in the diagram) allow for access by content and the formation of associative structures through rememorization under the control of other primaries (see Fig. 7). Periodic rememorization under the control of the original reference neuron also allows for stabilization of the memory trace, which means that the scheme accounts for both long and short term memory on the basis of the same fundamental mechanism. the reference to primary loading and calling processes must be mediated by temporal patterns of pulses (i.e. by codes). In this case the dendrite must contain a number of independent switching elements (which we identify with receptors), with the switching involving conformational changes at the molecular level. The theory imposes a number of functional requirements on the receptors. The most important of these are:

(i) The conformation records the occurrence of impulses or particular impulse sequences.

(ii) A regulation site controls the competence to be loaded by the incoming pulse or sequence of pulses, e.g. switches the molecule into the loadable state when an antidromic process is set up by the initial input. A fixation process, which follows loading, prevents the receptor from being unloaded by new input sequences.

(iii) The receptor must have an active site capable of catalyzing events leading to irnpulse formation.

The justification for the reference neuron scheme (and also the switching mechanisms which it implies) ultimately derive from its biological consequences. Again we defer these to the next section. Here, however, we should point out that the model allows for the stability of the memory trace by rememorizationmediated duplication of molecular conformations; for once the receptors are loaded, the same apparatus responsible for this loading can be used for other receptors in the same dendrite. In other words, reference neurons can periodically reload those primaries which respond to them. This is important, for it means that the model is capable of accounting for long, short, and very short term memory in terms of a single mechanism, with the only difference being that short term reference neurons do little or no reloading. Important memories can thus be transferred from short to long tel:m storage by rememorization under the control of long term reference neurons. In principle, they could also be assimilated directly into long term storage. 


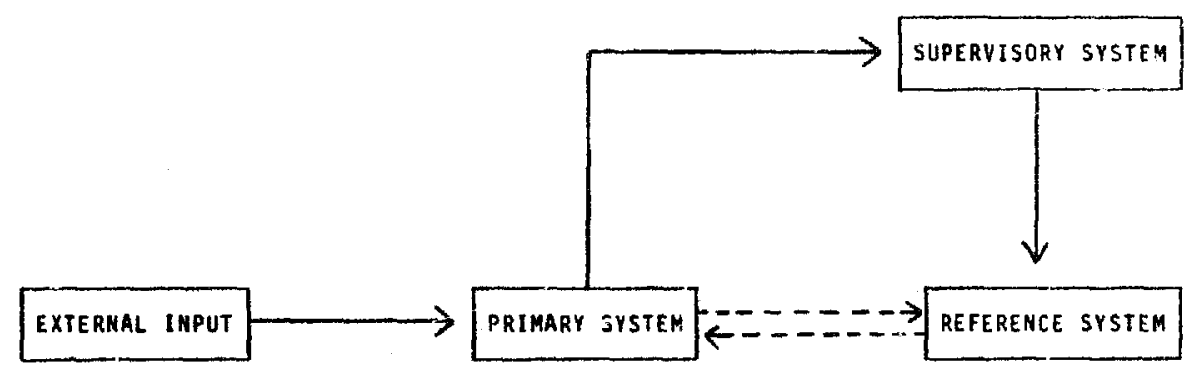

Fig. 7. Flow of information in the reference neuron scheme. The primary system of neurons includes all those neurons which fire in response to an exteenal input. Some of these are primary neurons proper, i.e. become sensitized when they fire and in such a way that they can be loaded and later called by neurons of the reference system. Neurons of the primary system (not necessarily primary neurons proper) may also load and subsequently call reference neurons, allowing for accessing of a complete memory in response to only some of the features of the original input. All "loading" and "calling" inputs are represented by dotted lines. The scheme requires a system of supervisory neurons to control the accessing of the reference neurons. For example, suppose that the supervisory system inhibits primary to reference inputs. Then reference neurons will activate one another in sequence, with resulting time ordered acquisition or recall. A second possibility is that the level of inhibition is decreased and control is given to the most highly excited reference neuron. This results in the content ordered structure. The third possibility is that more than one reference neuron is allowed to fire but some of the lower level neurons which mediate the connections between these reference neurons and primaries are suppressed. In this case it is possible to rememozize the resulting pattern of primary excitation under the control of still another reference neuron, thereby producing an associative structure. Rememorization provides the fundamental and general mechanism of memory manipulation in the reference neuron scheme.

\subsection{Interfaced model and interpretation brain structures}

The selection circuit model, with its enzymatic networks, accounts for the ability of the brain to compute the class of functions computable by finite automata, and to compute them with high efficiency. The reference neuron scheme adds to this the capability of rapid memory acquisition, manipulation, and retrieval. Moreover, the memories may be "scenic" memories, with the complexity limited only by the number of primaries reachable by reference neurons and by the pattern recognition abilities of the system.

The interfaced model is schematized in Fig. 8a. The topology of the diagram is the most economical possible from the standpcint of reducing the amount of neural tissue. Thus the reference neurons send their outputs (via party line neurons) through a horizontal layer of "telephone wires" into which the dendrites of the party line neurons dip. This arrangement, which serves to maximize the number or reference to primary contacts, is in fact found in the cerebral cortex (cf. Fig. 8b), where macroneurons of the inner five layers send apical dendrites into the outer, horizontal layer. The input itself comes primarily from the more interior regions of the brain and the output is channeled into the white matter, or inner layer of axons. The macroneurons themselves consist of a number of complexly interconnected types, perhaps the most prominant of which, from the standpoint of their apical dendrites, are the pyramidals. Thus it is reasonable to suppose that these correspond to primaxies. The cortex also consists of numerous microneurons. Since these have short axons and short dendrites, they cannot possibly be contacted, in any direct manner, by the reference neurons. Because of their large numbers and modulating roles they are more likely to be excitase based neurons of the selection circuit scheme, though pyramidals and other macroneurons could also be excitase based. Neurons of the brain are typically closely associated with glial cells. Since there is some evidence of transfer of RNA from glial cells to neurons (Hýden, 


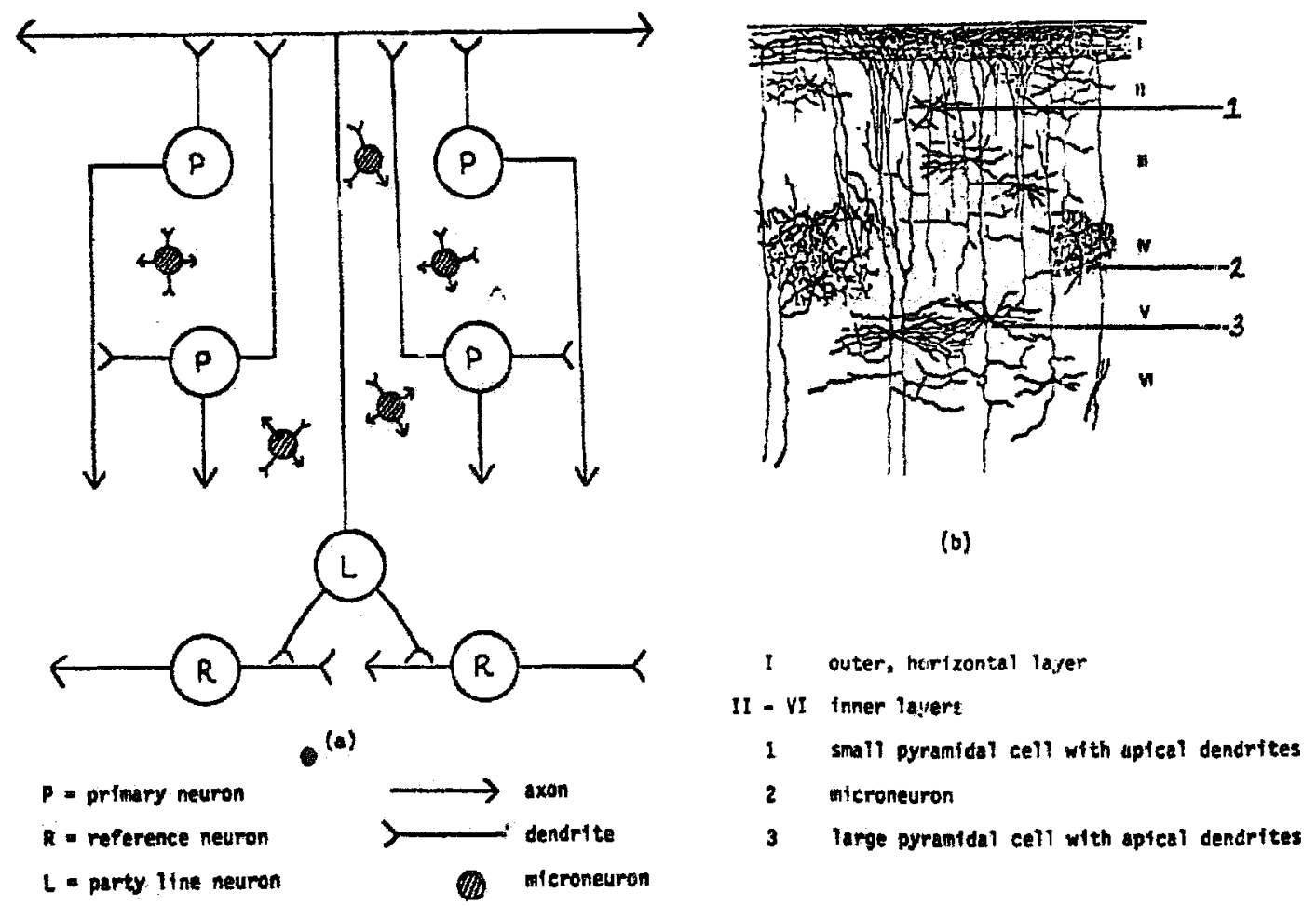

Fig. 8. Party line principle and histology of the cerebral cortex. (a) is the same as the simple network of Fig. 6, except for the addition of a party line (for economizing reference to primary contacts) and also some small, modulating neurons. The amount of neural tissue is minimized if the party line runs up and then horizontally along the top layer, thereby allowing easy access to upreaching dendrites of the primaries. The resulting organization provides an interpretation of the structure of the cerebral cortex. This is indicated in (b), which is a composite of rat and mouse Golgi preparations. The outer, horizontal layer corresponds to layer I of the cortex and the primaries correspond to pyramidals and other large cells of the inner five layers (not all of which are represented in (a)). The key point is that the (apjcal) dendrites of these large cells in fact extend into the horizontal layer. The cortex is also inter ispersed with microneurons (also schematically indicated in (a)), whose processes are too short to contact reference neurons and which therefore could not be the basis of memory in the reference heuron scheme. However, their large numbers (in humans), small size, and modulating role make them excellent candidates for excitase based neurons of the selection circuit system. The selection circuit centers (evaluation system, growth control), the supervisory system, and probably the reference neurons are presumably located in more ancient regions of the brain (as learning and memory predate the great expansion of the cortex). Notice that the use of party lines allows a single primary dendrite to be contacted by more than one reference neuron, so that neural codes must be used to distinguish the calls from these different reference neurons. This is why it is necessary to identify dendritic alterations with conformation changes of individual receptors. (Fig. 8(b) relabeled from Chang, 1951, by percisssion; cf. also Eccles, 1953, for more detailed identification of structures.)

1967a), and also because of a likely ontoge. netic relation of glial cells to cells of the immune system, it is reasonable to suppose that they are the actual site of transformation and that the transferred RNA is excitase RNA. The growth control, which regulates the pro. duction for export of excitase nucleic acid, and also the output evaluator would be expected to be located in phylogenetically more ancient regions of the brain.
Notice that it is the apical dendrites which serve as the "tape", i.e. as the locus of the conformational changes which form the physical basis of the memory trace. The remainder of the system, including the neurons to which these apical dendrites belong, correspond to the Turing automaton.

A similar interpretation is possible for the cerebellum, but without party lines. The basic structure is now the granule cell-parallel fiber- 
Purkinje cell circuit (cf. Llinas and Hillman, 1969). The branching of numerous granule cells gives a " $T$ "-structured (parallel fiber) cutput into which the dendrites of large Purkinje cells extend. The Purkinjes, which give rise to the only output, receive input (basically one-to-one) from climbing fibers and also external input (through the granule cells and therefore parallel fibers) from offshoots of an inner layer of horizontally running mossy fibers. These are thus the natural candidates for primaries, while granule cells, because of their large numbers and parallel fiber output are the natural candidates for the reference neurons. Actually the outputs ramify only about two $\mathrm{mm}$, impiying that storable and retrievable primary fatterns would have to be fairly localized. Higher in the phylogenetic scale the basic circuit is complicated by the addition of new types of interneurons, including Golgi cells (in the granular layer) and also outer stellate and basket cells. Like the Purkinjes, the Golgi ceils extend dendrites into the parallel fibers. Given their wide input and inhibitory influence on the granule cells, they are reasonable candidates for supervisory neurons (for the relation between inhibition and supervision see Fig. 7). Any neuron of the cerebellum is potentially excitase controlled, including primaries. However, stellate and basket interneurons, with their relatively short processes and inhibitory, modulating input on the Purkinjes are especially likely candidates for such control. The high homogeneity (and therefore the potential high redundancy) of the cerebellum is compatible with anci indeed a precondition for the selection circuit scheme. This potential redundancy is actually increased by the relative localization (in comparison to the cerebral cortex) of the primary patterns. Input from the growth control could be mediated by either mossy fibers or climbing fibers, but the latter are more likely because of their local excitatory influence and also because of their collateral excitatory influence on stellate and basket cells.

The above correlations between the ele- ments of the theory and structures of the cerebral corte: and cerebellum should be regarded as tentative and subject to experimental test. It is important that not all organism; or all parts of the brain which support learning and memory necessarily support both a reference neuron and selection circuit mechanism. Clearly the two mechanisms can operate independently and in some cases overlap in function. In general, the selection circuit mechanism is better for learning processes which require fine adjustment of motor control or sensory perseption. The reference neuron mechanism is better for rapid association of appropriate responses with appropriate stimuli (e.g. classical trial and error and instrumental leizning), or any type of learning or problem solving which depends on time-ordered, content-ordered, or associative deta structures (cf. Fig. 9). The cerebral cortex, with its pattern recognition, associative, and motor control functions would be expected to combine both types of learning, with the reference neuron mechanism subserving the formation and manipulations of associations, including associations between very different sense modalities, and the selection circuit mechanism adjusting firing patterns induced by the environment or concomitant to motor actions. In the case of the cerebellum the main function is apparently modulation of motor function (cf. Precht, 1974). The expectation therefore is that the selection circuit mechanism is especially important for fine tuning firing patterns and that the reference neuron mechanism allowis for an extremely short term memory and work space for fast calculation (cf. Conrad, 1976). Organisms in which the central nervous system is quite simple could not be expected to support both mechanisms. Thus a careful distinction must be made between modification and memory based leaming in experiments on such organisms. The distinction must also be made in systems in which both schemes are available since one or the other may be more important in any given situation. 


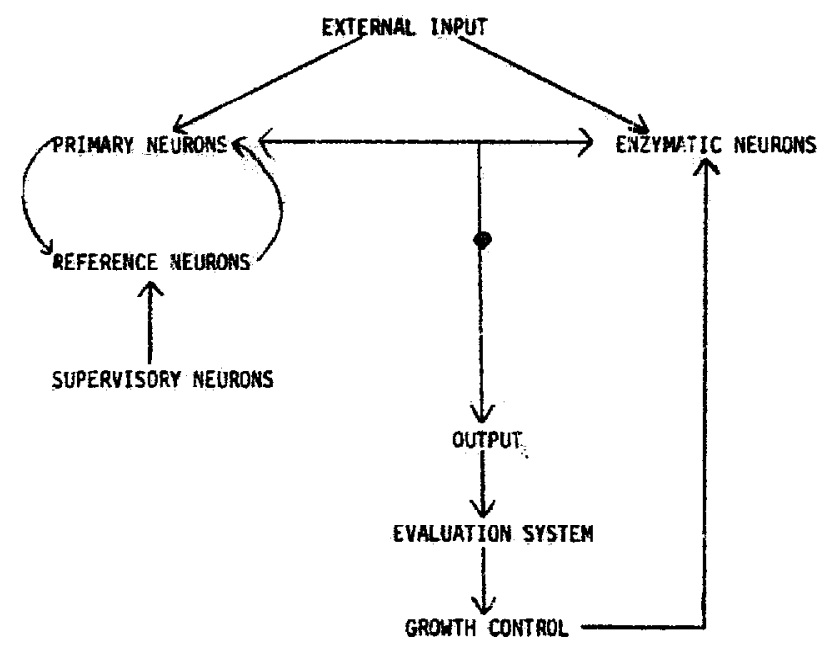

Fig. 9. Interfaced model. The diagram illustrates the flow of information in the combined scheme. The reference neuron mechanism (left) is responsible for storage and retrieval of manipulable memories, therefore for rapidly acquired memory, storage and retrieval of reference memories for reafference, associative processes such as classical conditioning and instrumental learning, formation of more general data structures (i.e. time-ordered, content-orclered, and associative structures) and imaginative processes involving restructuring of memory through rememorizaton. The selection circuit mechanism (right) is responsible for learning through gradual transformation of function in individual, enzymatic reurons or networks of such neurons, therefore for perceptual (or pattern recognition) and motor control (or pattern generation) learning and nore generally for leaming of intormation processes in which the high efficiency of enzymatic nets is critich. If the primary enzymatic neurons interact (or, in si ne cases, are identical) it is possible to gradually modify the firing patterns under the control of a given reference nellron and without disturbing the previously developed ceference Aeuron data structure. The two schemes are thus complementary, although potentially independent anô to some extent capable of overlapping function.

\section{Predictions of the theory}

The selection circuit and reference neuron schemes make a number of predictions. In what follows we describe the most important or testable of these.

\subsection{Chemical and molecular predictions}

Excitase nucleic acld can be used to transfer learned behavior from organism to organism, but with tfro provisions: 1) the learning must be modification-based and not memory-based; 2) the tissue between which the transfers take place must be essentially interchangeable. The second condition is consistent with the fact that interorganism transfers are in general only weakly effective or of disputed effectiveness (since such interchangeability would be the exception). It is also consistent with the fact that intraorganism transfers (from hemisphere to hemisphere) seem to be much more effective (Albert, 19i56; cf. Quarton, 1970). The effectiveness of transfers should also inerease with length of training period, since this allows for a latger and more adapted population of excitases. This also seems to be the case (McConnell et al., 1970). Conditions (1) and (2) are extremely restivictive and may therefore account for some of the discrepancy of result and discrepancy of conclusion surrounding transfer studies.

Since the selection circuits must ultimately stimulate or inhibit the produetion of excitase nucleic acid, their action must be mediated by enzymes whose activity or concentration changes during the learning process. As already meritioned, this is consistent with observations on specific changes in nuclear protein accompanying learning (Hýden, 1967b).

Thhibition of nucleic acid and protein synthesis would certainly be expected to interfere with or block modification-based learning. Such inhibitors will also affect memorybased learning because they prevent the synthesis of loadable receptor moleculas. The main predictions are: 1) inhibitors will have a marked effect on assimilation into long term memory since receptor molecules in dendrites contacting long term reference neurons are periodically reloaded and therefore in short supply; 2) they will have no marked or immediate effect on assimilation into short term memory because previously loaded riceptor molecules will continually become available for reuse. The predictions correspond to the key result of the biochemistry of memory, viz. that protein and nucleic acid inhibitors affect assimilation into long but not into 
short term memory (cf. Barondes, 1970; Agronoff, 1973).

\subsection{Anatomical and developmental predic- tions}

The selection circuit scheme requires redundancy and therefore implies a distributed character for modification-tiased learning. The reference neuron scheme, when interfaced, must also be redundant, frorn which it follows that memory and memory-based learning also have a distributed property. However, indiridual memories should also be accessible by stimulating specific: brain loci, viz. reference neurons, suitable primaries, or supervisory neurons. Thus the model is consistent with the dual distributed and localized character of the memory trace (cf. Lashley, 1929; Penfield and Perot, 1963).

The selection circuit scheme requires interehangeability of mutually trans 'ormable tissue and therefore at least statist cal homogeneity in the structure of such tissue. Thus the theory predicts that the brain is not plastic as regards any anatomical properties which affect the weighting coefficients, at least in parts of the brain which operate on the basis of the selection circuits. Aside trom this homogeneity requirement, however, the details of structure are entirely arbitrary; for once there is enough connectivity, any function can be implemented by intoducing the appropriate excitases.

Since the accumulation of modificationbased learning requires the accumulation of excitases and excitase nucleic asid, the learning process must be accompanied by increasing metabolic support. Since the glial cells are are the presumed site of transformation, it would be expected that these would assume some of the increased metabolic requirements, especially as they are isolated from the weighting coefficients. In fact, the number of glial cells does increase in the course of devel. opment and more markedly in enriched environments (Bennett et al., 19'70). In general plastic changes in the brain aye allowable so long as they are isolated from the weighting coefficients.

The reference neuron scheme also requires no particular details of connectivity, other than numerous reference to primary and primary to reference neuron contacts. The scheme is not affected by plastic changes at the synapse, e.g. in response to increased demands for firing, the only restriction being that such plastic changes do not affect the weighting coefficients of excitase based neurrons.

The general conclusion is thus that plastic changes in the brain are allowable so long as they are isolated from the weighting coefficients, but not otherwise. This is important, for it may account for the very diverse reports on plasticity versus specificity in the brain.

The theory makes general anatomical predictions in the sense that it requires the existence of selection circuits (including evaluation and growth control centers and connec. tions between the growth control and local, excitase governed networks) and also the existence of reference neuron circuits (including reference and primary neurons, with a definite, preferred topology for reference to primary contacts). Possible specific anatomical correlates of the theory have already been described in terms of functional interpretations for the structure of the cerebral cortex and cerebellum. The identification of the horizontal layer of cells in the cerebral cortex with party lines suggest that lesions cutting across this layer would have effects on the integrity of memory and the ability to assimilate mamories integrating different sense nodalities (assuming the number of lesions is sufficient to override redundancy in the reference to primary contacts). The predicted effects of undercutting are described further on in this section.

\subsection{Physiological and psychological predic- tions}

According to the selection circuit scheme, transformable neurons are capable of respond- 
ing to specific patterns of irput and therefore are potentially multithreshold elements. According to the reference neuron scheme, the dendrites of neurons which function as primaries are sensitizable and modifiable. Unfortunately, however, it seems difficult to test these features of the model directly, in part because of the difficulty of defining the input to neurons in the brain and in part because of the difficulty of recording from the microneurons.

The anatomical redundancy and arbitrariness of the details of connectivity in both schemes imply that the rate of both modification- and memory-based learning should decrease with cortical ablation. This is consistent with the so-called mass action laws of Lashley (1929). However, lesions to either the selection circuits themselves (i.e. the growtin control or evaluation system) or to the supervisory system in the reference neuron scheme should have radical effects on the learning process.

The theory gives a fairly simple account (on the basis of the reference neuron scheme) of classical conditioning and instrumental learning. For example, suppose that primaries firing in response to ringing are loaded by some reference neuron. Soon thereafter primaries firing in response to food (and therefore innately associated with salivation) are loaded by a second reference neuron. If the food is important to the organism, the supervisory system causes the second reference neuron to be loaded by the primaries called by the first, thereby establishing an associative structure. Instrumental (or classical trial and error) learning is explainable in the same way, except that the response is not innate. This mechanism allows for one exposure learning, the correct direction of association, diminishing retums (since the number of new loadings decreases with each repetition), extinction (since sensitized reference neurons are not reloaded by primaries which they do not call), and interference (since multiple bindings make the decision making process more difficult for the supervisory system). It also accounts for the importance of motivation since the probability of forming an association depends on the number of supervisory neurons activated by the supervisory system and also on the decision to reactivate the orignal reference neuron. These are all fundamental properties of classical conditioning and instrumental learning (cf. Miller, 1967).

The mechanism described above implies that undercutting the region associated with the conditioned stimulus (e.g. ringing) should prevent binding to the response (e.g. salivation), but that such binding would not be affected by vertical cuts. The reason is that primaries associated with ringing must load the reference neuron associated with salivation, which involves a vertical connection and no significant horizontal contacts are required in this case. This prediction agrees with experiment (cf. Campbell, 1965). Also, notice that the ease with which the stimulus is bound to the response depends on the number of primaries in the stimulus region which contact reference neurons which themselves contact primaries in the response region. The mechanism is thus consistent with and suggests an explanation for constraints on learning which apparently play an important role in some organisms.

Any memory scheme should be compatible with the well known principle of reafferance (that changes arising from the organism's own movements are subtracted from changes in the environment or, in some cases, from anticipated changes in the environment, cf. Von Holst and Mittelstädt, 1950; MacKay, 1962). This is clearly the case with the reference neuron scheme, since this provides a simple (perhaps the simplest) mechanism for accessing a reference scene either for comparison to current input or for computation prior to such comparison. However, the computations themselves (e.g. subtraction, anticioation) are not determined or constrained by the reference neuron apparatus but are a matter of the organization of the primary system, e.g. the inhibition or excitation of primary neurons resulting from the superposition of the refer- 
ence pattern of excitation with the environmentally induced patterm and also the way in which these patterns are coded into the motor outputs.

Perhaps the most important psychological feature of the theory is the distinction between modification- and memory-based learning. Situations involving one of these types of learning will lead to very different experimental conclusions than those involving the other, and at all levels of analysis, ranging from the chemical to the behavioral. Classical conditioning and instrumental learning, as well as their generalizations, belong to the memory-based category. However, the same effect could in many cases be achieved by modification-based learning, except that it would be very unlikely for this to arise in one exposure. Modification-based learning is better for motor control and perceptual learning, as previously discussed. The two types of learning may operate by themselves, but they may also contribute to a single learning process. For example, an association may be learned rapidly by the memory-based mechanism, but then the pattern recognition or motor control elements of this association may be gradually perfected by the modification-based mechanism. The two systems are thus complementary (cf. Fig. 9).

\section{Conclusions}

The problem of brain is particularly amenable, indeed perhaps only amenable, to the constructive method of analysis. This method depends first on the characterization of function and then on the economical design of systems which perform this function. In the present case we characterized function in terms of the Turing scheme, i.e. a finite automaton and work space which it manipu, lates. The selection circuit scheme, with its enzymatic networks, is the implementation of the finite automaton, with the provision that it fulfill the extra functional requirement of gradual modifiability. The reference neu- ron scheme adds to this the memory space capability, thereby giving the interfaced model the capabilities of a bona fide (molecular) computer.

Actually, we have something more than an ordinary computer, for an ordinary computer does not have the gradual modifiability property; nor are conventional neural networks of reasonable size sufficiently powerful to handle the global memory structures which are so easy to assimilate and retrieve in the reference neuron scheme.

The question may arise as to the criteria for an "economical" construction. Clearly the construction must be highly amenable to phylogenetic evolution, i.e. likely to arise and predominate in the course of Darwinian competition. In the case of the brain this means that the constructions should not involve improbable patterns of connectivity, unreasonable metabolic requirement, or biological processes which are not already used or derivable by gradual modification from other processes which the organism already uses. The selection circuit scheme fulfills the first criteria, for the connectivity is arbitrary; what is not arbitrary are only the enzymes which exploit this connectivity. Similarly, the connectivity of the reference neuron scheme is arbitrary within certain broad constraints. Neither scheme makes unreasonable metabolic demands and both of them operate on the basis of ubiquitous molecular principles, viz. specificity (in the case of the excitases of the selection circuit scheme) and conformation change (in the case of the receptors of the reference neuron scheme). The selection circuit scheme also involves elements of a regulative theory (e.g. enzyme induction or other control over enzyme activity) to mediate the action of the selection circuits on the production of exportable excitase nucleic acid.

Is it justifiable to base such constructive models on a neuron concept which seems so radically different from the classical concept? The basic physical insight behind the Hodgkin-Huxley and subsequent models of the neuron is that the neuron is a systam away 
from thermodynamic equilibrium and that ihe nerve impulse is essentially a manifestation of triggered return to equilibrium (cf. Hodgkin, 1964). In the selection circuit model we regard the excitase as being involved in the triggering process, which therefore allows the trigger to be modifiable. In the reference neuron scheme the trigger depends on the dendrite-bound receptor molecule, so that it is controllable. Such controllability and modifiability does not contradict the classical picture of impulse propagation and neurotransmission derived from studies of the peripheral nervous system, nor are they contradicted by their nonoccurrence in the peripheral nervous system (for their information processing function depends entirely on larger systems of circuitry in the brain). The neuron concept developed here is thus in some sense a generalization of the classical concept. The ultimate justification for this generalization derives from the experimental accuracy of its consequences, which so far have excellent agreement with the known facts. The same cannot be said for the narrower concept. To date this has been incapable of accounting for the main fact, viz. the ability of the brain to leam and remember.

\section{Acknowledgement}

Part of this work was done at the Institute for Information Seiences, University of Tübingen and at the Department of Biology, City College of the City University of New York.

\section{Reforences}

Agranoff, B.W., R.E. Davis, J.J. Brink, 1966, Chemical studies on memory fixation in goldfish, Brain A.s. 1, 303

Agranoff, B.W., R.E. Davis, L. Casola and R. Lin, 1967, Actinomycin D blocks formation of memory of shock avoidance in goldfiah. Science 158 , 1600 .
Agranoff, B.W., 1970, Recent studies on the stages of memory formation in goldfish, in: Molecular Approaches to Learning and Memory, W.L. Byrne (ed.) (Academic Press, New York).

Agranoff, B.W., 1973, Biochemical approaches to learning and memory, in: Macromolecules and Behavior, G.M. Ansell and P.B. Bradleyr (eds.) (MacMillan, London):

Albert, D.J., 1966, Memory in mammals: evidence for a system involving nuclear ribonucleic acid, Neuropsychology 4, 79.

Arbib, M., 1965, Brains, Machines and Mathematics (McGraw-Hill, New York).

Barondes, S.H. and H.D. Cohen, 1967, Comparative effects of cycloheximide and puromycin on cerebral protein synthesis and consolidation of memory in mice, Brain Res. 4, 45.

Barondes, J.H., 1970, Some critical variables in studies of the effect of inhibitors of protein synthesis on memory, in: Molecular Approaches to Learning and Memory, W.L. Byrne (ed.) (Academic Press, New York).

Bennett, E.L., M.R. Rosenzweig, M.C. Diamond, 1970 , Time courses of effects of differential experiences on brain measures and behavior in rats, in: Molecular Approaches to Learning and Memory, W.L. Byrne (ed.) (Academic Press, New York).

Bremermann, Rogson and Saloff, 1966, Global properties of evolution processes, in: Natural Automata and Useful Simulations, Pattee Edelsack, Fein and Callahan (eds.) (Spartan Books, Washington, D.C.).

Bremermann, H.J., 1967, Quantitative aspects of goal-seeking, selforganizing systems, Progr. Theor. Biol. 1, $\mathbf{5 9}$.

Bremermann, H.J., 1973, Artificial intelligence, pattern recognition and neurobiology, in: Physical Principles of Neuronal and Organismic Behavior, M. Conrad and M. Magar (eds.) (Gordon and Breac!, London).

Burks, A.W., B.P. Ziegler, R.A. Liang, J.H. Holland, 1974, Biologically mot vated automaton theory and automaton motivated biological research, in: Proceedings of the 1974 Conference on Biologically Motivated Automata Theoly (Institute of Electrical and Electronics Engineers, New York).

Byrne, W.L. (with 22 other a uthors), 1963, Memory transfer, Science 153, 658.

Byrne, W.L. (ed.), 1970, Molecular Approaches to Learning and Memory (Acadennic Press, New York).

Campbell, H.J. 1965, Correlative Playsiology of the Nervous System (Academic Press, New York).

Chang, H.T., 1951, Dendritic potential of cortical neurons produced by direct electrical stimulation of the cerebral cortex, J. Neurophysiol. 14,1.

Cohen, H.D., 1970, Learning, memory and metabolic inhibitors, in: Melecular Mechanisms of Learning 
and Memory, G. Ungar (ed.) (Plenum Press, New York).

Conrad, M., 1972a, Information processing in molecular systems. Currents in Modern Biology (Biosystems), Vol. 5, No. 1, 1.

Conrad, M., 1972b, The importance of molecular hierarchy in information processing, in: Towards a Theoretical Biology, Vol. 4, C.H. Waddington (ed.) (Edinburgh University Press, Edinburgh).

Conrad, M., 1973a, Is the brain an effective computer? Intern. J. Neurosci. 5, 167.

Conrad, M., $1973 \mathrm{~b}$, Molekulare Informationsverarbeitung in dem ZNS, in: Jahrestagung 1973 der Deutschen Gesellschaft für Biophysik, Kurzfassungen der Vorträge. (Jülich).

Conrad, M., 1974a, The limits of biological simulation, J. Theor. Biol. 45, 585.

Conrad, M., 1974b, Evolutionary learning circuits, J. Theor. Biol. 46, 167.

Conrad, M., 1974c, Computing in the biological mode. SIAM Newr, 7, No. 4.

Conrad, M., 1974d, Molecular information processing in the central nervous system, part I: selection circuits in the brain, in: Physics and Mathematics of the Nervous System, M. Conrad, W. Güttinger and M. Dal Cin (eds.) (Springer, Heidelberg).

Conrad, M., $1974 \mathrm{e}$, Molecular information processing in the central nervous system, part II: molecular data struciures, in: Physies and Mathematics of the Nervous System, M. Conrad, W. Guittinger and M. Dal Cin (eds.) (Springer, Heidelberg).

Conrad, M., 1974f, Molecular autonata, in: Physics and Mathematics of the Nervous System, M. Conrad, W. Gittinger and M. Dal Cin (eds.) (Springer, Heidelberg).

Conrad, M., 1975a, Molecular information structures in the brain, to appear in Journal of Neuroscience Research.

Conrad, M., $1975 \mathrm{~b}$, Conformational model of memory and memory manipulation (Abstract), Biophys. J. 15, No. 2, Part II, 117a.

Conrad, M., 1976, Constructive molecular model of cerebellar function. To appear in Proc. Fifth Pittsburg Conf. on Modeling and Simulation.

Conrad, M. and M. Dal Cin, 1972, Notes on automata, semigroups and computation. Center for Theoretical Studies Lecture Note Series CTS-LN-1. 72.

Conrad, M., W. Güttinger, and M. Dal Cin (eds.), 1974, Physics and Mathematics of the Nervous System (Springer, Heicelberg).

Davis, M., 1958, Computability and Unsolvability (McGraw-Hill, New York).

Eccles, J.C., 1953, The Neurophysiological Rasis of Mind (Oxford University Press, Oxford).

Teigenbaum, E. and J. Feldman, 1963, Computers and Thought (McGraw-Hill, New York).
Gibts, M.E. and R.F. Mark, 1973, Inhibition of Memory Formation (Plenum Press, New York).

Glassman, E., 1969, The biochemistry of learning: an evaluation of the role of RNA and protein, Ann. Rev. Biochem. 38, 605.

Hodgkin, A.L., 1964, The Conduction of the Nerve Impulse (Liverpool University Press, Liverpool).

Hýden, H., 1967a, RNA in brain cells, in: The Neurosciences, G.C. Quarton, T. Melnechuk and F.O. Schmitt (eds.) (Rockefeller University Press, New York).

Hýden, H., 1967b, Biochemical changes accompany ing learning, in: The Neurosciences, G.C. Quarton, T. Melnechuk and F.O. Schmitt (eds.) (Rockefeller University Press, New York).

Hýden, H. and P.W. Lange, 1971, Time sequence analysis of proteins in brain stem, limbic system and cortex during training, Biochim. Biol. Sper. 9, 275.

Jerne, N.K., 1955, The natural selection theory of antibody formation, Proc. nat. Acad. Sci. U.S., 41, 849.

John, E.R., 1967, Mechanisms of Memory (Academic Press, New York).

Josephson, B.D., 1974, The artificial intelligence/psychology approach to the study of the brain and nervous system, in: The Physics and Mathematics of the Nervous System, M. Conrad, W. Güttinger and M. Dal Cin (eds.) (Springer, Heidelberg).

Katchalsky, A. and E. Neumann, 1973, Hysteresis and molecular memory record, in: The Physicai Principles of Neuronal and Organismic Behavior, M. Conrad and M. Magar (eds.) (Gordon and Breach, itondon).

Lashley, K.S., 1929, Brain Mechanisms and Intelligence (Chicago University Press, Chicago). (Reprinted 1963 by Dover, New York.)

Lehninger, A., 1970, Mitochondria and their neurofunction, in: The Neurcsciences: a Second Study Program, F.O. Schmitt (ed.) (Rockefeller University Press, New York).

Llinás, R. and D.E. Hillman, 1969, Physiological and morphological organization of the cerebellar circuits in various vertebrates, in: Neurobiology of Cerebellar Evolution and Development, R. Llinás (ed.) (Amer. Med. Ass Educ. and Res. Fed., Chicago).

Mackay, D.M., 1962, Theoretical mocels of space perception, in: Aspects of the Theor of Artificial Intelligence, C.A. Muses (ed.) (Plenum Press, New York).

McConnell, H.M., 1970, Molecular motion in biological membrane, in: The Neuroscienres: a Second Study Program, F.O. Schinitt (ed.) (Rockefeller University Press, New York).

McConnell, J.V. and J.M. Shelby, 1970, Memory transfer experiments in invertebrates, in: Molecu. 
lar Mechanizms in Memory and Learning, G. Un. gar (ed.) (Plenum Press, New York).

McConnell, J.V., T. Shigehisn and H. Salive, 1970, Attempts to transfer approach and avoidance response by RNA injections in rats, in Molecular Approaches to Learning and Memory, W.L. Byrne (ed.) (Academic Press, New York).

McCulloch, W.S. and W. Pitts, 1943, A logical calculus of the ideas immanent in nervous activity. Bull Máth. Biophys. 5, 115.

Miller, N.E., 1967, Certain facts of learning relevant to the search for its physical basis, in: The Neuroseiences, G.C. Quarton, T. Melnechuk and F.O. Schmitt (eds.) (Rockefeller University Press. New York).

Minsky, M., 1961, Steps toward artificial intelligence, Proc. IRE 49, 18.

Minsky, M, 1967, Computation: Finite and Infinite Machines (Prentice-Hall, Englewood Cliffs, N.J.).

Neumann, E., 1974, An integral physico-chemical model for bioexcitability, in: The Physics and Mathematics of the Nervous System, M. Conrad, W. Güttinger and M. Dal Cin (eds.) (Springer, Hejdelberg).

Penfield, W. and P. Perot, 1963, The brain's record of auditory and yisual experience: a final summary and discussion, Brain 86, 595.

Precht, W. 1974, Cerebellar morphology and physiology: introductory remarks, in: The Physics and Mathematics of the Nervous System, $\mathrm{Mi}_{\text {. Conrad, }}$ W. Güttinger and M. Dal Cin (eds.) (Springer, Heidelberg).

Quarton, G.C., 1970, The enhancement of learning by drugs and the transfer of learning by macromolecules, in: The Neurosciences, G.C. Quarton,
T. Melnechuk and F.O. Schmitt (eds.) (Rockefeller University Press, New York).

Rosen, R., 1969, Hierarchical organization in automata theoretic models of the central nervous sys. tem, in: Information Processing in the Nervous System, K.N. Leibovic (ed.) (Springer, Heidelberg).

Rosen, $R$, 1974, Hyteresie in sycreme with a sincle steady state, Center for Theoretical Biology preprint (Buffalo), to appear in Bull. Math. Biol.

Schmitt, F.O., 1970, Molecular neurobiology: an interpretive survey, in: The Neurosciences: Second Study Program, F.O. Schmitt (ed.)(Rockefeller University Press, New York).

Schwabauer, R., 1976, Enzymatic neurons, J. Theor. Biol. 59, 223.

Smith, C.E., 1962, Is memory matter of enzyme induction? Science 138,889 .

Stent, G.S., 1972, Prematurity and uniqueness in scientific discover: Scient. Amer. 227, No. 6, 84.

Turing, A.M., 1936 in omputable numbers with an application to tic Eischeidungtsproblem, Proc. London Math. Soc. \&r. 2, 42, 230.

Ungar, G. (ed.), 1970, Molecular Mechanisms in Learning and Memory (Plenum Press, New York).

Ungar, G., 1973, Molecular approaches to neural coding, in: The Physical Principles of Neuronal and Organismic Behavior, M. Conrad and M. Magar (eds.) (Gordon and Breach, London).

von Holst, E. and H. Mittelstädt, 1950, Das reafferenzprinzip, Naturwissenschaffen 37,464 .

Weiss, P.A., 1970, Neuronal dynamics and neuroplasmic flow, in: The Neurosciences: a Second Study Program, F.O. Schmitt (ed.) (Rockefeller University Press, New York). 